Psychotherapeut 2011 · 56:201-202

DOI 10.1007/s00278-011-0827-2

Online publiziert: 20. April 2011

(c) Springer-Verlag 2011

\author{
Alexandra Martin ${ }^{1}$ Jens Gaab ${ }^{2}$ \\ ${ }^{1}$ Psychosomatik, Psychotherapieforschung, Universitätsklinikum \\ Erlangen, Friedrich-Alexander Universität Erlangen-Nürnberg \\ ${ }^{2}$ Klinische Psychologie und Psychotherapie, Psychologisches Institut, \\ Universität Zürich, Schweiz
}

\title{
Chronische Erschöpfung
}

\section{Das unsichtbare Symptom?}

Erschöpfung ist eine der häufigsten Beschwerden in der Allgemeinbevölkerung und im Gesundheitssystem, entweder als Symptom einer somatischen Erkrankung oder einer psychischen Störung oder als Leitsymptom einer eigenständigen Störung. $\mathrm{Zu}$ unterscheiden sind akute, vorübergehende Erschöpfungszustände, die u. a. durch angemessene Erholungsphasen wieder abklingen, von andauernder, chronischer Erschöpfung. Die chronische Erschöpfung verursacht Leiden und Beeinträchtigung, weil sie umfassend ist und genau das ver- bzw. behindert, was das Leben (zu großen Teilen jedenfalls) ausmacht: Aktivität. Chronische Erschöpfung verursacht auch hohe Kosten, sowohl individuelle als auch gesellschaftliche. Chronische Erschöpfung ist ein Problem; sie ist nicht ortbar, nicht spezifisch, nicht direkt messbar und schlecht kommunizierbar.

Wenn chronische Erschöpfung also häufig, beeinträchtigend und bedeutsam ist, warum ist dann chronische Erschöpfung so unsichtbar? Im Unterschied zu anderen, in vielerlei Hinsicht vergleichbaren, Störungen und Symptomen - man denke nur an chronische Schmerzen fehlen (im deutschsprachigen Raum jedenfalls) eine angemessene Betrachtung und Auseinandersetzung mit der chronischen Erschöpfung. Das Ziel dieses Themenhefts ist es, dem eine Übersicht von Beiträgen und Themen entgegenzusetzen und zu zeigen, dass die chronische Erschöpfung nicht unsichtbar bleiben sollte (oder darf?).

Bei dem chronischen Erschöpfungssyndrom (",chronic fatigue syndrome“, CFS) stehen andauernde, organisch nicht hinreichend erklärbare, körperliche und/ oder mentale Erschöpfungssymptome im Vordergrund des Beschwerdebilds. In zwei Übersichtsbeiträgen wird auf aktuell diskutierte ätiologische Konzepte des CFS eingegangen. Die „Bedeutung des Stresses bei chronischer Erschöpfung" wird von Nater vorgestellt. Mitgeteilt werden die Befunde zu Stressbelastung bei Personen mit CFS, die die Basis für die Diskussion ihrer ätiologischen Relevanz darstellen. $\mathrm{Zu}$ Recht - meinen wir - trägt diese Arbeit den Zusatz „Psychobiologischer Ansatz", da die Interaktion aus psychischen und körperlichen Faktoren und nicht die alleinige Betrachtung eines dieser Faktoren betont wird. Befunden zufolge sind Stressbelastung und traumatische Erfahrungen bei CFS-Patienten erhöht. In Verbindung gebracht werden diese Beobachtungen mit Befunden der nachhaltigen Beeinträchtigung der endokrinen Stressachse, die eine Erklärung für die Vulnerabilität zur Ausbildung chronischer Erschöpfungszustände darstellen könnte. Neben prospektiver Forschung in diesem Bereich wird es wichtig sein, die Spezifität dieser Mechanismen für CFS weiter zu beleuchten.

In der anschließenden Diskussion von Gaab zum Thema „Somatische Ursache des chronischen Erschöpfungssyndroms" wird auf historisch relevante ätiologische Konzepte und ganz aktuelle Befunde zu einer Virusbelastung (aktueller Kandidat der Kontroverse ist das „Xenotropic murine leukemia virus-related virus") bei CFS-Betroffenen eingegangen. Anhand dieser Darstellung wird deutlich, wie gerade bei organisch nicht hinreichend er- klärbaren Körperbeschwerden die Suche nach somatischen Ursachen als „Messlatte" für die Legitimisierung der Beschwerden wird - und zwar nicht nur aus Sicht der Betroffenen, sondern oftmals auch der Behandler.

Bei diversen somatischen Krankheiten stellt Erschöpfung nicht nur ein Begleitsymptom, sondern durchaus ein wesentliches, behandlungsbedürftiges Problem dar. So wird beispielsweise zwischenzeitlich der Fatigue-Belastung bei Tumorerkrankungen, multipler Sklerose und "Human-immunodeficiency-virus“(HIV-)Infektionen größere Bedeutung in Forschung und Behandlung beigemessen. Die Originalie von Kuhnt et al. stellt eine Untersuchung zu Prädiktoren tumorassoziierter Fatigue dar. Die Ergebnisse legen nahe, die Diagnostik und Behandlung von akuter Fatigue frühzeitig zu berücksichtigen, um einer möglichen Chronifizierung vorzubeugen.

Eine weitere Manifestation des Erschöpfungserlebens wird von Gawlick u. Reck aufgegriffen - Erschöpfung im Rahmen von Depression. Bekannt ist, dass Personen, die unter Depressionen leiden, oftmals medizinische Hilfe aufgrund begleitender körperlicher Probleme aufsuchen, wie beispielsweise aufgrund von Erschöpfung, Antriebslosigkeit, körperlicher Schwäche oder Kopfschmerzen. Weniger Aufmerksamkeit hat demgegenüber bislang die Relevanz von Erschöpfung und Depression im Rahmen der Schwangerschaft erhalten. Genau dies wird von den Autorinnen thematisiert. Die psychotherapeutische Behandlung der Depression bekommt hier eine zusätzliche Bedeu- 
tung aufgrund der Abwägung von Nutzen und Kosten einer psychopharmakologischen Behandlung, hier gemeint v. a. im Hinblick auf ihre potenzielle Auswirkung auf den Fetus.

Der Psychotherapie von Erschöpfungszuständen sind zwei weitere Beiträge gewidmet. Einerseits wird dargestellt, wie die Evidenzbasierung von Psychotherapie bei organisch unklarer Erschöpfung ist (Martin u. Gaab), andererseits wird therapeutisches Vorgehen im Rahmen einer stationären Therapie anhand eines Behandlungsfalls exemplifiziert (Weimer u. Kraus). Zu den bislang international am besten abgesicherten Therapien des CFS zählt die kognitive Verhaltenstherapie, während andere Psychotherapieformen bei diesem Beschwerdebild kaum evaluiert wurden (s. aktuelle Ergebnisse aus Metaanalysen und Originalstudien bei Martin u. Gaab). Demgegenüber sind die psychotherapeutischen Konzepte bei Fatigue im Rahmen von Tumorerkrankungen oder multipler Sklerose durchaus variabler - und in der therapeutischen Praxis finden oftmals Kombinationen aus verschiedenen therapeutischen Verfahren und Methoden statt. Dies wird schließlich über die Darstellung eines stationären Behandlungsproblems von Weimer u. Kraus verdeutlicht, das sich an Personen mit einer Burn-out-Problematik richtet.

Bewusst zielen die Beiträge darauf ab, Erschöpfung bei verschiedenen Krankheitsbildern - d. h. im Rahmen des CFS, bei Tumorerkrankungen, bei depressiven Störungen sowie im Rahmen des Burnout-Konzepts - zu thematisieren. Psychotherapeutische Strategien wurden bei allen diesen Varianten erprobt und stellen oftmals die einzige Behandlungsvariante dar.

Muss die chronische Erschöpfung unsichtbar sein? Nein. Es gibt chronische Erschöpfung, und es gibt Möglichkeiten, diese zu erkennen, $\mathrm{zu}$ thematisieren und v. a. damit umzugehen.

\section{Korrespondenzadressen}

\section{Prof. Dr. Alexandra Martin}

Psychosomatik, Psychotherapieforschung, Universitätsklinikum Erlangen, FriedrichAlexander Universität Erlangen-Nürnberg Schwabachanlage 6, 91054 Erlangen

Alexandra.Martin@uk-erlangen.de

\section{PD Dr. Jens Gaab}

Klinische Psychologie und Psychotherapie, Psychologisches Institut, Universität Zürich Attenhoferstr. 9, 8032 Zürich, Schweiz j.gaab@psychologie.uzh.ch
Schwerpunktthemen

Das Herausgebergremium der Zeitschrift Psychotherapeut lädt Autorinnen und Autoren ein, an den geplanten Schwerpunkten mitzuarbeiten und geeignete Manuskripte einzureichen. Diese werden dem üblichen Reviewverfahren unterzogen. Darüber hinaus freuen wir uns über die Zusendung freier (unaufgeforderter) Originalia zu selbstgewählten Themen.

Bitte schicken Sie Ihren Beitrag an die Redaktion:

Regine.Karcher-Reiners@springer.com

Die Schwerpunktplanung ist vorläufig und kann kurzfristig umdisponiert werden.

Heft 6/11: Psychotherapie und elektronische Medien

(Deadline 26.7.2011)

Heft 1/12: Migration (Deadline: 17.9.2011)

Heft 2/12: Vergessen - Erinnern Gestalten (LPW 2011)

(Deadline 18.11.2011)

Heft 3/12: Neurowissenschaften (Deadline 14.1.2012)

Heft 4/12: Psychoedukation (Deadline 28.2.2012)

Heft 5/12: Bedeutung des Therapeuten für den Therapieerfolg (Deadline 30.4.2012) 